\title{
Circulating microRNAs as minimally invasive biomarkers for cancer theragnosis and prognosis
}

\author{
William C. S. Cho* \\ Department of Clinical Oncology, Queen Elizabeth Hospital, Kowloon, Hong Kong
}

\section{Edited by:}

Michael Rossbach, Genome Institute of Singapore, Singapore

Reviewed by:

Haruhiko Siomi, Keio University School of Medicine, Japan

Dong Xu, University of Missouri, USA

${ }^{*}$ Correspondence:

William C. S. Cho, Department of Clinical Oncology, Queen Elizabeth Hospital, Kowloon, Hong Kong. e-mail:chocs@ha.org.hk
Novel cancer biomarker discovery is urgently needed for cancer theragnosis and prognosis, and among the many possible types of samples, blood is regarded to be ideal for this discovery as it can be collected easily in a minimally invasive manner. Results of the past few years have ascertained the quantification of microRNA (miRNA) as a promising approach for the detection and prognostication of cancer. Indeed, an increasing number of studies have shown that circulating cancer-associated miRNAs are readily measured in plasma or serum and they can robustly discriminate cancer patients from healthy controls, as well as distinguishing between good-prognosis and poor-prognosis patients. Furthermore, recent findings also suggest the potential of circulating miRNAs in the screening, monitoring, and treatment of cancer. This article summarizes the most significant and latest discoveries of original researches on circulating miRNAs involvement in cancer, focusing on the potential of circulating miRNAs as minimally invasive biomarkers for cancer theragnosis and prognosis.

Keywords: blood-based biomarker, cancer, circulating microRNA, minimally invasive biomarker, molecular tumor marker, oncomir, prognosis, theragnosis

\section{INTRODUCTION}

In terms of suffering and death, cancer is tragic yet partly preventable. The developments of valid biomarkers to prevent, detect, and treat this devastating disease can save millions of lives. However, the establishment of clinically validated biomarkers for cancer remains an insurmountable task despite the advances in molecular biology. An ideal tumor marker should be measured easily, reliably, and costeffectively using a minimally invasive assay with high analytical sensitivity and specificity. Results of the last few years have ascertained the quantification of microRNA (miRNA) as a promising approach for the detection and prognostication of cancer (Cho, 2007, 2010a). Nevertheless, it remains uncertain whether circulating miRNA is tumor specific and whether systemic miRNA analysis has utility in cancer management. Indeed, miRNA is present in human blood in a remarkably stable form protected from endogenous RNase activity (Mitchell et al., 2008). An increasing number of studies have shown that circulating cancer-associated miRNAs (oncomirs) are readily measured in plasma or serum and they can robustly discriminate cancer patients from healthy controls. This article summarizes the most significant and latest findings of original researches on circulating miRNAs involvement in cancer, focusing on the potential of circulating miRNAs as minimally invasive biomarkers for cancer theragnosis and prognosis (Table $\mathbf{1}$ ).

\section{MiRNA AND CANCER}

MicroRNAs are emerging as important modulators in cellular pathways, and they appear to play a key role in tumorigenesis (Cho, 2010b). Numerous studies have documented the implications of miRNAs in nearly every carcinogenesis process, including tumor development, apoptosis, invasion, and metastasis, as well as anticancer drug resistance (Cho, 2009, 2010c).

\section{ROLE OF CIRCULATING MIRNAS IN THE MOLECULAR PATHOGENESIS AND PROGRESSION OF CANCER}

Previous study has suggested that profiling of circulating miRNAs may help identify promising biomarkers of various pathologic conditions (Ji et al., 2009). In the study of chronic lymphocytic leukemia (CLL), low expression of $m i R-29 b, m i R-29 c, m i R-181$ family, and $m i R-223$ were found to be strongly associated with disease progression in CLL cases harboring $17 \mathrm{p}$ deletion, whereas high expression of miR-181a in those harboring trisomy 12 suggested more aggressive disease. These biomarkers may be clinically useful to assess the tumor behavior in CLL (Visone et al., 2009). A pilot study also evaluated the circulating miRNAs associated with tumor progression in breast cancer patients. It provided evidence that the relative concentration of $m i R-155$ in serum significantly discriminated primary breast cancer patients from healthy women. Within the primary breast cancer cohort, patients at advanced tumor stages had significantly higher $m i R-34 a$ than patients at early tumor stages. In the metastatic patients, miR-10b, miR-34a, and miR-155 correlated with the presence of overt metastases (Roth et al., 2010).

\section{DIAGNOSTIC AND PROGNOSTIC VALUE OF CIRCULATING mIRNAs FOR CANCER}

The releases of miRNAs from malignant cells in body fluids are candidate diagnostics for a variety of cancers. A recent study reported that the release of miRNAs from breast cancer cells into blood, milk, and ductal fluids was selective and that the selection of released miRNAs might correlate with malignancy. In particular, the bulk of miR-451 and miR-1246 produced by malignant mammary epithelial cells was released into the blood, but the majority of these miRNAs produced by non-malignant mammary epithelial cells was 


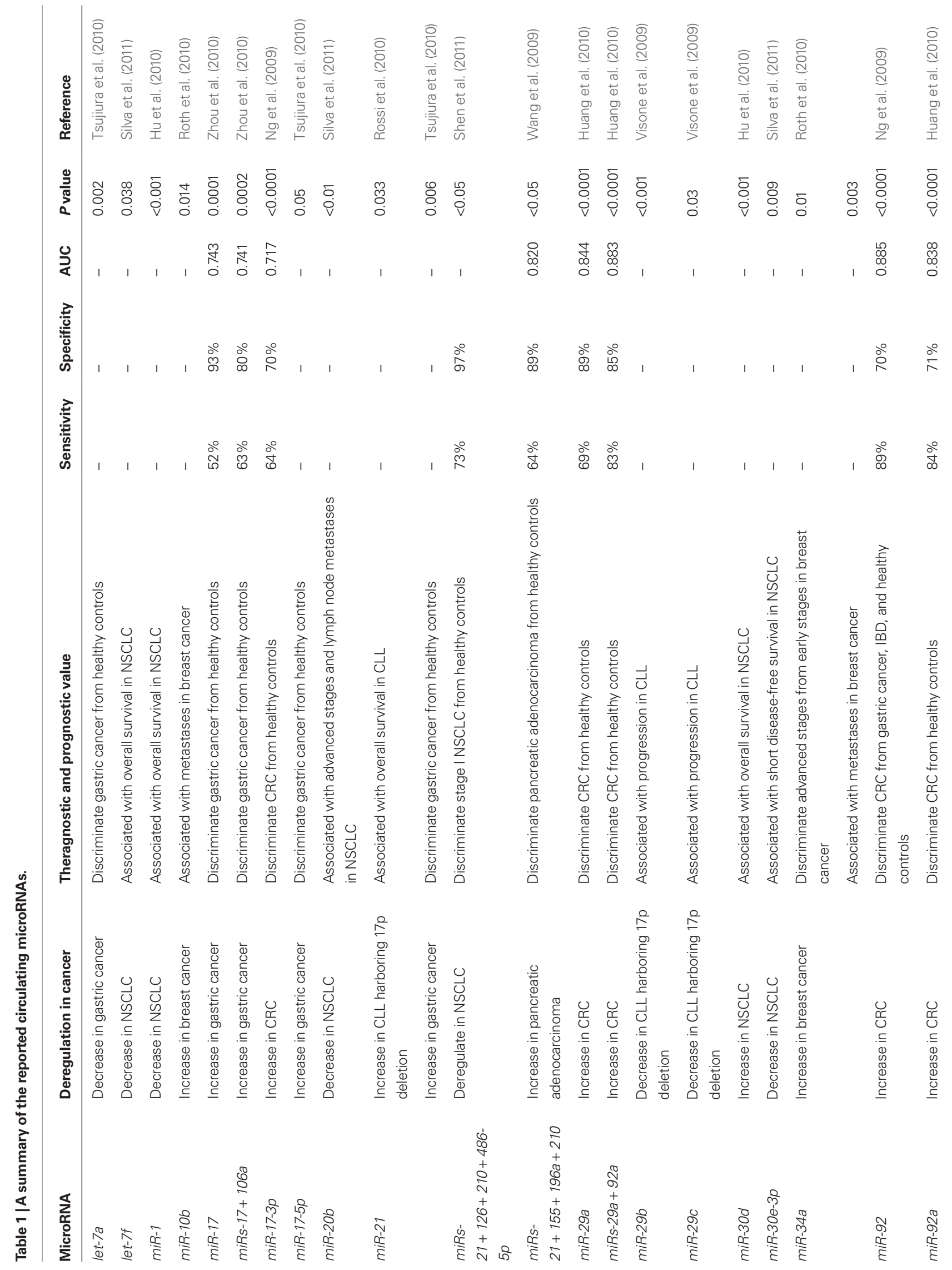




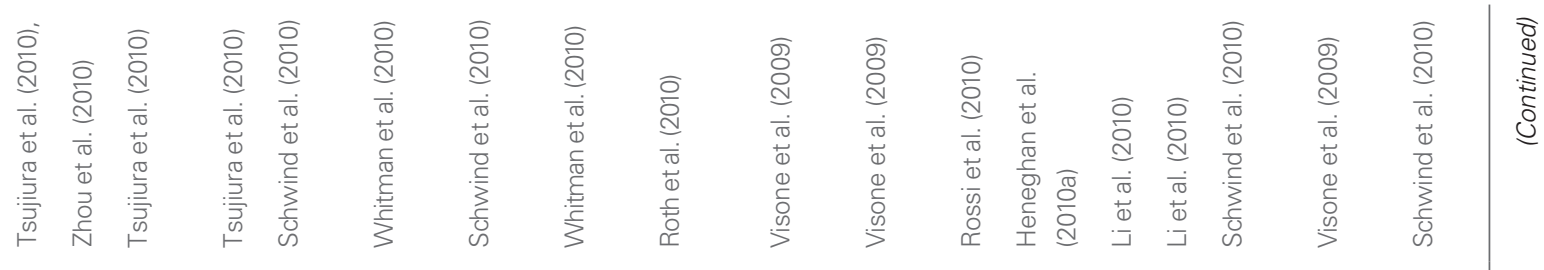

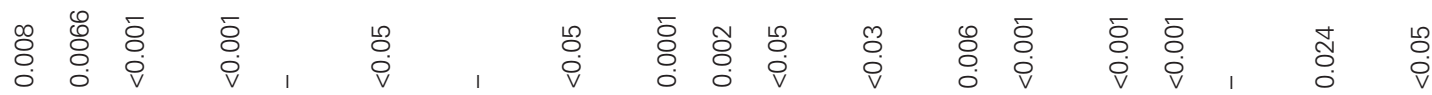

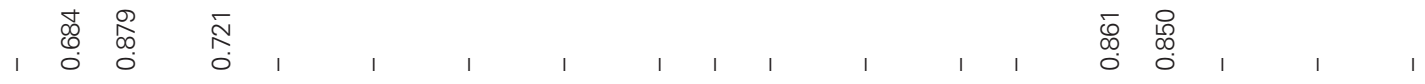

1 离

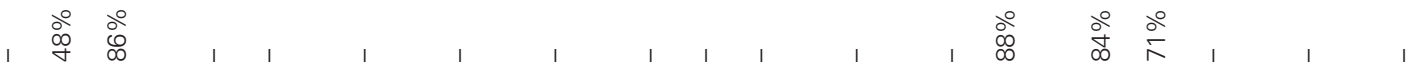

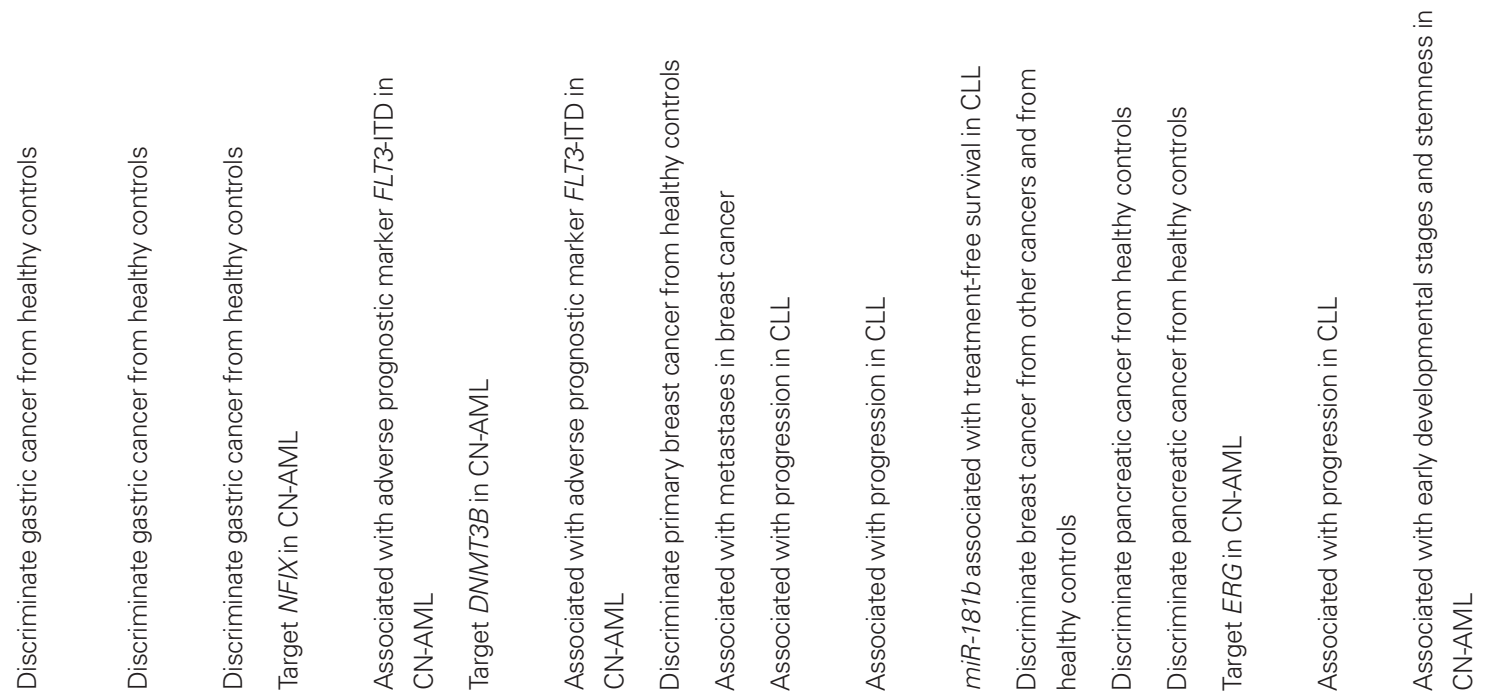

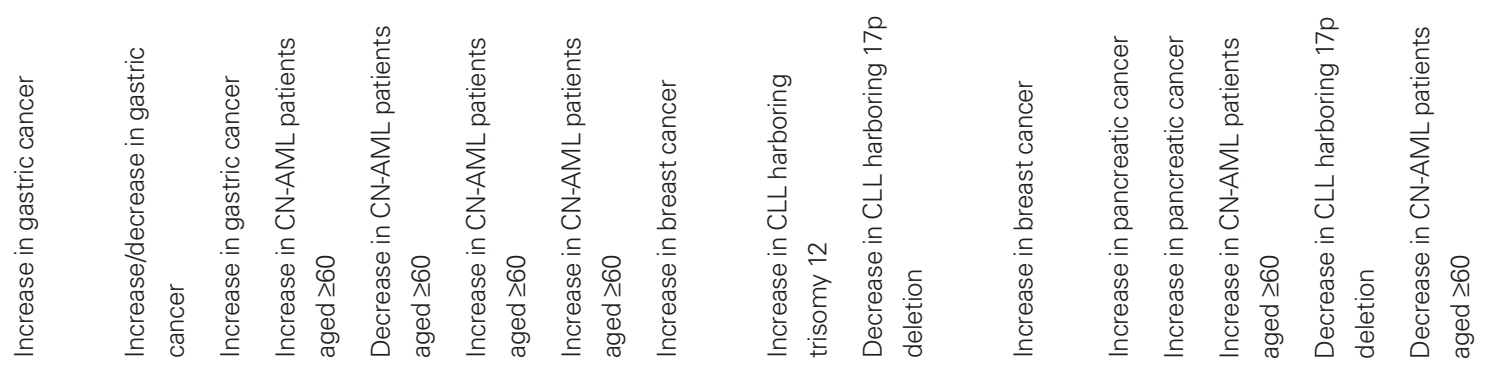

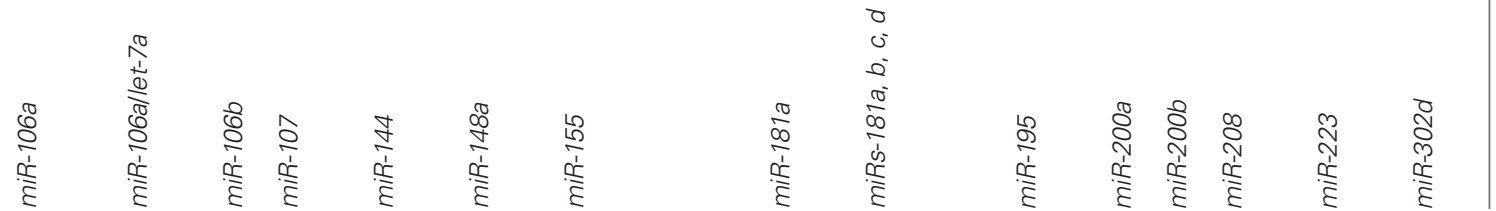


retained. Their findings suggest that the selective release of miRNA is an important consideration for the identification of circulating miRNA as molecular tumor marker (Pigati et al., 2010).

An investigation of plasma miRNAs in colorectal cancer (CRC) indicated that $m i R-29 a$ and $m i R-92 a$ could significantly discriminate neoplasia from healthy controls, and combined analyses using these two miRNAs revealed higher sensitivity and specificity. These data suggest that plasma $m i R-29 a$ and $m i R$-92a have strong potential as minimally invasive biomarkers for the detection of CRC (Huang et al., 2010). Another study found that plasma miR-92 also had significant diagnostic value for CRC. This biomarker could significantly differentiate CRC from gastric cancer, inflammatory bowel disease, and normal subjects, suggesting it to be a potential minimally invasive molecular marker for CRC diagnosis (Ng et al., 2009).

In the analysis of serum miRNAs, most pancreatic cancers displayed hypomethylation and over-expression of miR-200a and miR-200b, silencing of SIP1 by promoter methylation, and retention of E-cadherin expression. The elevated serum levels of miR-200a and $m i R-200 b$ in most patients with pancreatic cancer may have diagnostic utility (Li et al., 2010). On the other hand, profiling of $m i R-21, m i R-155, m i R-196 a$, and $m i R-210$ showed that miRNA profiling in plasma could also differentiate pancreatic adenocarcinoma patients from healthy controls. These results show the feasibility of developing plasma miRNA profiling as a sensitive and specific blood-based biomarker assay for pancreatic cancer that has the potential of translation to the clinic with additional improvements in the future (Wang et al., 2009). Similarly, the concentrations of $m i R-21, m i R-17-5 p, m i R-106 a$, and $m i R-106 b$ were significantly higher in gastric cancer patients than healthy controls, whereas let-7a was lower in gastric cancer patients. The levels of aberrantly expressing miRNAs were also significantly reduced in post-operative samples than pre-operative controls. These findings suggest that detection of circulating miRNAs may provide new complementary tumor markers for gastric cancer (Tsujiura et al., 2010).

The identification of a patient who is prognostically good or poor is very important for the development of effective treatment approach (Cho, 2011a). Recent findings have revealed a great potential of circulating miRNA signatures as molecular fingerprints to predict survival of cancer patients. Genome-wide serum miRNAexpression analysis found that the levels of four miRNAs ( $m i R-1$, $m i R-30 d, m i R-486$, and $m i R-499$ ) were significantly associated with the overall survival of non-small cell lung cancer (NSCLC) patients. These four serum miRNA signatures may serve as an independent minimally invasive predictor for the overall survival of NSCLC (Hu et al., 2010). Moreover, the evaluation of plasma miRNAs also detected decreased levels of let-7f, $m i R-20 b$, and $m i R-30 e-3 p$ in the vesicles of NSCLC patients than healthy controls. The plasma levels of let-7f and $m i R-30 e-3 p$ were associated with overall survival and short disease-free survival, respectively. These results suggest that plasma vesicle-related miRNAs obtained by minimally invasive methods can serve as circulating tumor biomarkers of discriminating and prognostic values (Silva et al., 2011). On the other hand, quantitative reverse transcription-polymerase chain reaction (qRT-PCR) analysis in CLL patients with chromosome 17p deletion also found that $m i R-21$ expression levels were significantly higher in patients with poor overall survival, whereas lower miR-181b 
expression levels significantly predicted treatment-free survival. A 21FK score ( $m i R-21$ qRT-PCR, fluorescence in situ hybridization, Karyotype) was developed to stratify patients according to overall survival and it was found to be a useful tool for distinguishing between good-prognosis and poor-prognosis CLL patients (Rossi et al., 2010).

\section{POTENTIAL OF CIRCULATING MIRNAs IN SCREENING, MONITORING, AND TREATMENT OF CANCER}

Cancer is often diagnosed at a late stage with concomitant poor prognosis. Developing minimally invasive biomarkers that can diagnose cancer, particularly at an early stage, may improve its outcome. Evaluated by qRT-PCR, a panel of four plasma miRNAs ( $m i R-21, m i R-126, m i R-210$, and $m i R-486-5 p$ ) yielded $86 \%$ sensitivity and $97 \%$ specificity in distinguishing NSCLC patients from the healthy controls. Furthermore, the panel of miRNAs produced $73 \%$ sensitivity and $97 \%$ specificity in identifying stage I NSCLC patients. These results confirm that altered expressions of the miRNAs in plasma may provide potential blood-based biomarkers for NSCLC at an early stage (Shen et al., 2011).

To determine whether circulating miRNAs were tumor specific, a panel of oncomirs in the whole blood of pre-operative cancer patients (melanoma, breast, colon, prostate, and renal cancers) were assessed. Elevated circulating $m i R-195$ was found to be breast cancer specific and it could significantly differentiate breast cancer from other cancers and from healthy controls (Heneghan et al., 2010a). Furthermore, the circulating levels of $m i R-195$ and let-7a decreased in breast cancer patients post-operatively to levels comparable with healthy controls (Heneghan et al., 2010b). These findings suggest that circulating miRNAs have potential use as breast cancer biomarkers for early stage disease and they may also prove to be useful in clinical management during the peri-operative period. In fact, the detection of occult cancer cells in peripheral blood has recently received a great deal of attention regarding the prediction of postoperative cancer recurrence and for novel strategies of adjuvant therapy. In the peripheral blood samples from post-operative gastric cancer patients, the levels of miR-17 and miR-106a were significantly higher than those in healthy controls. These results indicate that the detection of miRNAs in peripheral blood may also be a tool for monitoring circulating tumor cells in patients with gastric cancer (Zhou et al., 2010).

\section{REFERENCES}

Cho, W. C. (2007). Oncomirs: the discovery and progress of miRNAs in cancers. Mol. Cancer 6, 60.

Cho, W. C. (2009). Role of miRNAs in lung cancer. Expert Rev. Mol. Diagn. 9, 773-776.

Cho, W. C. (2010a). MiRNAs: potential biomarkers for cancer diagnosis, prognosis and targets for therapy. Int. J. Biochem. Cell Biol. 42, 1273-1281.

Cho, W. C. (2010b). MiRNAs in cancer from research to therapy. Biochim. Biophys. Acta 1805, 209-217.

Cho, W.C. (2010c). MiRNAs as therapeutic targets for lung cancer. Expert Opin. Ther. Targets 14, 1005-1008.
Cho, W. C. (2010d). "Omics approaches in cancer research," in An Omics Perspective on Cancer Research, ed. W. C. Cho (Berlin: Springer), 1-9.

Cho, W.C. (2011a). Molecular diagnostics for monitoring and predicting therapeutic effect in cancer. Expert Rev. Mol. Diagn. 11, 9-12.

Cho, W. C. (2011b). MiRNAs in Cancer Translational Research. Berlin: Springer.

Cho, W.C. (2011c). Grand challenges and opportunities in deciphering the role of non-coding RNAs in human diseases. Front. Genet. 2:1. doi: 10.3389/ fgene. 2011.00001

Heneghan, H. M., Miller, N., Kelly, R., Newell, J., and Kerin, M. J. (2010a).

In primary cytogenetically normal acute myeloid leukemia (CN-AML) patients aged 60-69 years, FLT3-internal tandem duplication (ITD) was found to be significantly associated with overall survival and shorter disease-free survival. It was revealed that FLT3-ITD-associated miRNA-expression signatures included over-expressed $m i R-155$, as well as under-expressed $m i R-144$ and miR-451. These miRNA-expression signatures may provide biologic insights for novel therapeutic approaches in older CN-AML patients with molecular high risk (Whitman et al., 2010). Conversely, low $B A A L C$ and $E R G$ expression levels were identified to be associated with better outcome in older CN-AML patients aged $\geq 60$ years. $H O X$ genes and $H O X$-gene-embedded miRNAs were up-regulated in low BAALC expressers, whereas low ERG expressers presented with up-regulation of miR-148a which targeted DNMT3B. These miRNA-expression signatures may aid in identifying new targets and novel therapeutic strategies for older patients with low BAALC and $E R G$ expressions (Schwind et al., 2010).

\section{CONCLUSION}

MicroRNA is a cutting-edge topic in the scientific and medical fields, the identification of oncomirs as blood-based biomarkers proceeds at a fast pace (Cho, 2011b). Numerous promising developments have been elucidated using omics technologies in cancer research (Cho, 2010d). The applications of microarray, microfluidics, nanofluidics, next generation sequencing, qRT-PCR, and bioinformatics have enabled the discoveries of a number of circulating miRNAs as potential biomarkers for cancer theragnosis and prognosis. These bloodbased biomarkers have a revolutionary impact on cancer research over recent years. A number of circulating miRNAs have been found to be promising molecular tumor markers for early detection or survival prediction, some were even revealed to be involved in cancer progression or metastasis. The prospect for circulating miRNAs as minimally invasive biomarkers for cancer is excellent, although there are some challenges that the researchers have to conquer before these small non-coding RNAs can be fully understood and utilized (Cho, 2011c). With the accessibility of large sample sets, the range of technologies available, and the increasing evidences that there is a signature of changes derived by cancer in blood which may contribute to theragnosis and prognosis, all suggest that circulating miRNAs, perhaps accompanying other markers, will become widely used minimally invasive biomarkers for cancer in the future.

Systemic miRNA-195 differentiates breast cancer from other malignancies and is a potential biomarker for detecting noninvasive and early stage disease. Oncologist 15, 673-682.

Heneghan, H. M., Miller, N., Lowery, A. J., Sweeney, K. J., Newell, J., and Kerin, M. J. (2010b). Circulating microRNAs as novel minimally invasive biomarkers for breast cancer. Ann. Surg. 251, 499-505. Hu, Z., Chen, X., Zhao, Y., Tian, T., Jin, G., Shu, Y., Chen, Y., Xu, L., Zen, K., Zhang, C., and Shen, H. (2010). Serum microRNA signatures identified in a genome-wide serum microRNA expression profiling predict survival of non-small-cell lung cancer. J. Clin. Oncol. 28, 1721-1726.
Huang,Z., Huang, D., Ni, S., Peng,Z., Sheng, W., and Du, X. (2010). Plasma microRNAs are promising novel biomarkers for early detection of colorectal cancer. Int. J. Cancer 127, 118-126.

Ji, X., Takahashi, R., Hiura, Y., Hirokawa, G., Fukushima, Y., and Iwai, N. (2009). Plasma miR-208 as a biomarker of myocardial injury. Clin. Chem. 55, 1944-1949.

Li,A., Omura, N., Hong, S. M., Vincent, A., Walter, K., Griffith, M., Borges, M., and Goggins, M. (2010). Pancreatic cancers epigenetically silence SIP1 and hypomethylate and overexpress miR$200 \mathrm{a} / 200 \mathrm{~b}$ in association with elevated circulating miR-200a and miR-200b levels. Cancer Res. 70, 5226-5237. 
Mitchell, P. S., Parkin, R. K., Kroh, E. M., Fritz, B. R., Wyman, S. K., PogosovaAgadjanyan, E. L., Peterson, A., Noteboom, J., O’Briant, K. C., Allen, A., Lin, D. W., Urban, N., Drescher, C. W., Knudsen, B. S., Stirewalt, D. L., Gentleman, R., Vessella, R. L., Nelson, P. S., Martin, D. B., and Tewari, M. (2008). Circulating microRNAs as stable blood-based markers for cancer detection. Proc. Natl. Acad. Sci. U.S.A. 105, 10513-10518.

Ng, E. K., Chong, W. W., Jin, H., Lam, E. K., Shin, V. Y., Yu, J., Poon, T. C., Ng, S. S., and Sung, J. J. (2009). Differential expression of microRNAs in plasma of patients with colorectal cancer: a potential marker for colorectal cancer screening. Gut 58, 1375-1381.

Pigati, L., Yaddanapudi, S. C., Iyengar, R., Kim, D. J., Hearn, S. A., Danforth, D., Hastings, M. L., and Duelli, D. M. (2010). Selective release of microRNA species from normal and malignant mammary epithelial cells. PLoS ONE 5, e13515. doi: 10.1371/journal. pone. 0013515

Rossi, S., Shimizu, M., Barbarotto, E., Nicoloso, M. S., Dimitri, F., Sampath, D., Fabbri, M., Lerner, S., Barron, L. L., Rassenti, L. Z., Jiang, L., Xiao, L., Hu, J., Secchiero, P., Zauli, G., Volinia, S., Negrini, M., Wierda, W., Kipps, T. J., Plunkett, W., Coombes, K. R., Abruzzo, L. V., Keating, M. J., and Calin, G. A. (2010). MicroRNA fingerprinting of CLL patients with chromosome $17 \mathrm{p}$ deletion identify a miR-21 score that stratifies early survival. Blood 116 945-952.

Roth, C., Rack, B., Muller, V., Janni, W., Pantel, K., and Schwarzenbach, H. (2010). Circulating microRNAs as blood-based markers for patients with primary and metastatic breast cancer. Breast Cancer Res. 12, R90.

Schwind, S., Marcucci, G., Maharry, K., Radmacher, M. D., Mrózek, K., Holland, K. B., Margeson, D., Becker, H., Whitman, S. P., Wu, Y.Z., Metzeler, K. H., Powell, B. L., Kolitz, J. E., Carter, T. H., Moore, J. O., Baer, M. R., Carroll, A. J., Caligiuri, M.A., Larson, R. A., and Bloomfield, C. D. (2010). BAALC and ERG expression levels are associated with outcome and distinct gene and microRNA expression profiles in older patients with de novo cytogenetically normal acute myeloid leukemia: a cancer and leukemia group B study. Blood 116, 5660-5669.

Shen, J., Todd, N. W., Zhang, H., Yu, L., Lingxiao, X., Mei, Y., Guarnera, M., Liao, J., Chou, A., Lu, C. L., Jiang, Z., Fang, H., Katz, R. L., and Jiang, F. (2011). Plasma microRNAs as potential biomarkers for non-smallcell lung cancer. Lab. Invest. doi: 10.1038/labinvest.2010.194. [Epub ahead of print].

Silva, J., García, V., Zaballos, A., Provencio, M., Lombardía, L., Almonacid, L., García, J. M., Domínguez, G., Peña, C., Diaz, R., Herrera, M., Varela, A., and Bonilla, F. (2011). Vesiclerelated microRNAs in plasma of
NSCLC patients and correlation with survival. Eur. Respir. J. doi: 10.1183/09031936.00029610. [Epub ahead of print].

Tsujiura, M., Ichikawa, D., Komatsu, S., Shiozaki, A., Takeshita, H., Kosuga, T. Konishi,H., Morimura, R., Deguchi, K., Fujiwara, H., Okamoto, K., and Otsuji, E. (2010). Circulating microRNAs in plasma of patients with gastric cancers. Br. J. Cancer 102, 1174-1179.

Visone, R., Rassenti, L. Z., Veronese, A. Taccioli, C., Costinean, S., Aguda, B. D., Volinia, S., Ferracin, M., Palatini, J., Balatti, V., Alder, H., Negrini, M., Kipps, T. J., and Croce, C. M. (2009). Karyotype-specific microRNA signature in chronic lymphocytic leukemia. Blood 114, 3872-3879.

Wang, J., Chen, J., Chang, P., LeBlanc, A. Li, D., Abbruzzesse, J. L., Frazier, M. L., Killary, A. M., and Sen, S. (2009). MicroRNAs in plasma of pancreatic ductal adenocarcinoma patients as novel blood-based biomarkers of disease. Cancer Prev. Res. (Phila.) 2 807-813.

Whitman, S.P., Maharry,K., Radmacher,M. D., Becker, H., Mrózek, K., Margeson, D., Holland, K. B., Wu, Y. Z., Schwind, S., Metzeler, K. H., Wen, J., Baer, M. R., Powell, B. L., Carter, T. H., Kolitz, J. E. Wetzler, M., Moore, J. O., Stone, R. M. Carroll, A. J., Larson, R. A., Caligiuri, M A., Marcucci, G., and Bloomfield, C. D. (2010). FLT3 internal tandem duplication associates with adverse outcome and gene- and microRNA-expression signatures in patients 60 years of age or older with primary cytogenetically normal acute myeloid leukemia: a cancer and leukemia group B study. Blood 116, 3622-3626.

Zhou, H., Guo, J. M., Lou, Y. R., Zhang, X. J., Zhong, F. D., Jiang, Z., Cheng, J., and Xiao, B. X. (2010). Detection of circulating tumor cells in peripheral blood from patients with gastric cancer using microRNA as a marker. J. Mol. Med. 88, 709-717.

Conflict of Interest Statement: The author declares that the research was conducted in the absence of any commercial or financial relationships that could be construed as a potential conflict of interest.

Received: 03 January 2011; accepted: 11 February 2011; published online: 28 February 2011

Citation: Cho WCS (2011) Circulating microRNAs as minimally invasive biomarkers for cancer theragnosis and prognosis. Front. Gene. 2:7. doi: 10.3389/ fgene.2011.00007

This article was submitted to Frontiers in Non-Coding RNA, a specialty of Frontiers in Genetics.

Copyright (C) 2011 Cho. This is an openaccess article subject to an exclusive license agreement between the authors and Frontiers Media SA, which permits unrestricted use, distribution, and reproduction in any medium, provided the original authors and source are credited. 